\title{
Quality Adjustment in Non-spanning Markets with Variety Aversion
}

\author{
Kazuhiko Nishimura
}

Received: 17 February 2013 / Accepted: 20 June 2013 / Published online: 9 July 2013

(C) 2013 K. Nishimura; licensee Springer. This is an Open Access article distributed under the terms of the Creative Commons Attribution License (http://creativecommons.org/licenses/by/2.0), which permits unrestricted use, distribution, and reproduction in any medium, provided the original work is properly cited.

\begin{abstract}
This paper examines the dynamic process of quality adjustment in cases where the economy lacks a sufficient number of markets for coordinating the level of attributes that configure the qualities of products. It shows that an adjustment process through the development and selection of commodities with different qualities may succeed in achieving efficiency or at least, meet the necessary conditions for efficiency. This is true if the user can identify with the altered product and is unaffected by variety in the commodity. It also holds true when the consumer assesses product variety in a smooth (differentiable) function, but not necessarily so if the assessment is non-smooth regarding the homogeneous state. An unidentifiable case can also be subject to inefficiency as the effort of a small quality adjusting agent becomes attenuated.
\end{abstract}

Keywords Quality adjustment · Adaptive selection · Trial and error · Variety averse $\cdot$ Non-spanning markets

JEL Classification $\mathrm{L} 15 \cdot \mathrm{H} 23 \cdot \mathrm{O} 33$

\section{Introduction}

Many transactions in a product supply chain involve quality coordinations among vertically differentiated intermediate commodities. For example, manufacturers of turbine engines are selective about the performance of turbine blades, whereas producers of the blades focus on cost effectiveness that is better quality blades at lower

K. Nishimura $(\bowtie)$

Faculty of Economics, Nihon Fukushi University, Mihama, Aichi 470-3295, Japan

e-mail: nishimura@n-fukushi.ac.jp 
costs. Similarly, the recycling industry will be affected by the recyclability of the secondary (recycled) inputs, which is controlled by the producers of primary products. The quality adjustment process described hereinafter is therefore somewhat in the same vein as the demand-driven technology evolution, as discussed in Adner and Levinthal (2001).

Based on the Lancasterian consumer theory (Lancaster 1966), quality is usually treated as the bundle of objective characteristics (attributes) of a commodity for which the consumer holds positive values. ${ }^{1}$ This paper is concerned with commodities that are vertically differentiated by objective quality: In other words, better quality is preferred by all users (consumers) if the commodities are sold at the same price. For example, PCs with a fast processor and a relatively slow processor are vertically differentiated, whereas chocolate and vanilla ice cream are horizontally differentiated because they are equally priced. ${ }^{2}$

Quality can be subject to externalities when product attributes lack their individual markets. Attributes generally do not retain the excludability property that a normal commodity has in theory due to their bundledness, rendering the independent coordination of attributes impractical. While excludability being one of the characteristic of a public good, Leland (1977) and Dreze and Hagen (1978) indicate that for quality choices, individuals' private assessment (i.e., the Lindahl price) of attributes are needed when the implicit market of attributes is incomplete. Conversely, these studies claim that if every attribute's implicit price spans the explicit competitive prices of goods, the (first-order) conditions for efficiency are met.

This study focuses on cases in which the market of attributes forms an incomplete, non-spanning structure, and thus quality is partially or completely external to the economic coordination system. Given these circumstances, the study posits that producers follow a quality adjustment process based on trial-and-error, that is, they adjust the quality in accordance with demand in order to predominate the product market with improved quality. The idea is parallel to previous studies on dynamic search processes, such as Callander (2011) under conditions of uncertainty and Sato (2012) for public goods. Nevertheless, it was Jwa (1985) who studied quality development in vertically differentiated products, assuming the price of quality being available.

Jwa (1985) also pointed out the reduction in variety by the nature of competitive equilibrium, resulting in a single homogeneous quality across products. Similar issues have been addressed in Nishimura (2008) about the quality of secondary materials in the recycling industry. More recently, from a slightly different perspectives, Brun and Pero (2012) analyzed a firm's decision to reduce its variety of industrial products. Such a reduction in the vertically differentiated variety is essentially equivalent to being variety averse, discussed in a number of empirical studies (e.g., Levav et al.

\footnotetext{
${ }^{1}$ A characteristics approach has been extensively applied in the marketing and innovation metrics literature, using methods ranging from hedonic functions (i.e., Rosen 1974) to discrete choice models (i.e., McFadden 1986).

${ }^{2}$ Horizontal product differentiation and the related models based on Hotelling (1929) have been widely discussed in industrial organizations literature (e.g., Economides 1984; Shaked and Sutton 1987, etc.).
} 
2010; Kaiser 2011). The essential problem is that products need differentiation to enable the selection of a better quality product, while it is refused to diversify after one being selected. ${ }^{3}$

In this paper, a model of quality adjusting process under non-spanning structure of implicit markets over the attributes is presented. Change in quality is controlled by the assumption that a competitive producer not only maximizes profit by taking the price of product, but also alters the quality (or that of the attributes) with the speculation that a product with a different quality (and corresponding price) can lead the market. The benchmark model deals with cases in which the buyer of a product is indifferent to the heterogeneity in product quality. The buyer chooses the quality altered product if the product with marginally altered quality is more valuable to him or her relative to the marginal cost of altering the quality, which the seller adds to the price of the product. ${ }^{4}$ The new product will then dominate the market, thereby improving the quality in the market.

The steady state of the benchmark process is shown to satisfy the necessary conditions of efficiency. However, this is not the case when heterogeneity in product quality matters to the buyer. For example, intermediate and recycling industries typically use bulky inputs for production, and thus consistent quality is often preferred. However, if the producer of an upper stream industry alternates the quality of its product, the lower stream industry would consequently face heterogeneous inputs. This study presents several cases of buyers' preference for homogeneous/heterogeneous quality. It shows that even when products with varied quality are differently priced, the quality adjustment procedure may not function when the variety assessment of the buyer is non-smooth around the center. Cases where heterogeneous products are assessed with a flat price could lead to inefficiency because the smaller the producer, the more its effort become attenuated.

The rest of the paper is organized as follows. Section 2 briefly specifies the spanning property in the implicit markets for attributes, and by doing so, focus on the equilibrium state of a non-spanning economy where some attributes are external to the economy. Section 3 outlines the foundations of the quality adjustment process driven by one of producers (a speculator) seeking alternating price-quality combinations in a non-spanning environment. Subsequently, the case of a consumer without distastes upon heterogeneous products is discussed. In Sect. 4, we consider cases where the consumer is variety averse, that is, the consumer/user prefers consistent product quality. Section 5 is devoted to demonstrating the results with a simple model of a recycling economy. Section 6 concludes.

\footnotetext{
${ }^{3}$ This contention is also addressed in bioevolution literature such as Batten and Boschetti (2008). Hosoda (2012) deliberately uses the linear joint production models instead.

${ }^{4}$ It is assumed that a producer may not observe the buyer's reservation price of quality, but may know its relativity with the asked price by observing the buyer's decision.
} 


\section{Market Structure}

\subsection{Spanning Economy}

We begin with a simple general equilibrium model of a Lancasterian economy that incorporates the essential features needed for the subsequent discussions. We use the term "commodities" to designate goods and services subject to actual market transactions. There are $N$ types of commodities, indexed $j=1, \ldots, N$ and $Z$ types of attributes indexed $i=1, \ldots, Z$. The quantity of $j$ commodity is represented by $x_{j}$, while the whole set of commodities is represented by a vector $\mathbf{x}=\left(x_{1}, \ldots, x_{N}\right)$. Similarly, the amount of $i$ attributes within the whole economy is represented by $s_{i}$ and the set of attributes is represented by $\mathbf{s}=\left(s_{1}, \ldots, s_{Z}\right) . a_{i j}$ denotes the amount of attributes $i$ per unit of commodity $j$, hence $s_{i}=\sum_{j} x_{j} a_{i j}$ by definition and $\mathbf{A}$ denotes the $N \times Z$ matrix with the typical entry being $a_{i j}$. Therefore, the quality of commodity $j$ can be represented by a vector of the quantity of attributes $\mathbf{a}_{j}=$ $\left(a_{1 j}, \ldots, a_{Z j}\right)$.

We assume a representative consumer with utility function $U(\mathbf{s})$ that maps from a given bundle of attributes $\mathbf{s}$ to a real number. Also, let $B$ denote the budget for the representative consumer and $\mathbf{p}=\left(p_{1}, \ldots, p_{N}\right)$ the price vector for commodities, where $p_{j}$ designates the price of the $j$ commodity. We specify the representative consumer's behavior as follows:

$$
\max _{\mathbf{s}, \mathbf{x}} U(\mathbf{s}) \quad \text { s.t. } \quad B=\mathbf{x p}^{\prime}, \quad \mathbf{s}=\mathbf{x A} .
$$

Ordinal assumptions for the utility function must be applied, that is, $U$ is strictly quasi-concave and at least twice continuously differentiable with respect to all entries. The first-order conditions for this optimization follow below:

$$
U_{\mathbf{s}} \mathbf{A}^{\prime}=\lambda \mathbf{p}
$$

where the scalar $\lambda$ designates the Lagrangian multiplier for the budget constraint. Note that a prime is used to denote transposition and we write

$$
U_{\mathbf{s}}=\left(\frac{\partial U(\mathbf{s})}{\partial s_{1}}, \ldots, \frac{\partial U(\mathbf{s})}{\partial s_{Z}}\right)
$$

and so forth to save space.

The firms are characterized by the transformation functions that maps from $\mathbf{x}$ into $\mathbf{s}$, that is, $\mathbf{G}(\mathbf{x})=\left(G_{1}(\mathbf{x}), \ldots, G_{Z}(\mathbf{x})\right)=\left(s_{1}, \ldots, s_{Z}\right)=\mathbf{s}$, and the production possibility set for the attributes $\mathbf{s}$, namely, $F(\mathbf{s})=0$. An implicit transformation function of the commodities can be constructed using these terms, that is, $F(\mathbf{G}(\mathbf{x}))=0$. A marginal firm will seek to earn profits under the transformation function: ${ }^{5}$

$$
\max _{\mathbf{x}, \mathbf{s}} \mathbf{x p}^{\prime} \quad \text { s.t. } \quad F(\mathbf{s})=0, \quad \mathbf{s}=\mathbf{G}(\mathbf{x}) .
$$

\footnotetext{
${ }^{5}$ The earned profits will be transferred to the representative consumer's budget $B$, and thus we have the closed system. 
The first-order conditions for this behavior is described below:

$$
\mu F_{\mathbf{s}} \mathbf{G}_{\mathbf{x}}^{\prime}=-\mathbf{p}
$$

where the scalar $\mu$ designates the Lagrangean multiplier for the transformation function. Also, note also that we write $\mathbf{G}_{\mathbf{x}}^{\prime}=\left\{\mathbf{G}_{x_{1}}, \ldots, \mathbf{G}_{x_{N}}\right\}$, where

$$
\mathbf{G}_{x_{j}}^{\prime}=\left(\frac{\partial G_{1}(\mathbf{x})}{\partial x_{j}}, \ldots, \frac{\partial G_{Z}(\mathbf{x})}{\partial x_{j}}\right) .
$$

At the efficient state of this simple Lancasterian economy it must be true that the representative consumer's marginal rate of substitution is tangent to the marginal rate of transformation with respect to all existing attributes. This is the familiar first-order condition for economic efficiency.

$$
\frac{U_{s_{1}}}{F_{s_{1}}}=\frac{U_{s_{2}}}{F_{s_{2}}}=\cdots=\frac{U_{s_{Z}}}{F_{s_{Z}}} .
$$

On the other hand, by Eqs. (2) and (4), we have the following conditions for the commodities market equilibrium:

$$
-\lambda \mu F_{\mathbf{s}} \mathbf{G}_{\mathbf{x}}^{\prime}=U_{\mathbf{s}} \mathbf{A}^{\prime}
$$

As it is evident from these equations, we need $\mathbf{G}_{\mathbf{x}}=\mathbf{A}$ and a right inverse matrix to exist, to assure that the commodities market equilibrium will reach a state of efficiency; however, this would be possible only if $N \geq Z$ and the matrix is non-singular. These conditions are otherwise called the spanning property (Leland 1977) of the implicit market for attributes.

\subsection{Model of Non-spanning}

Based on the above argument, we introduce a model for non-spanning in a most simplistic manner. In the model, there are only two commodities in the economy, one of which is identified by its first attribute, quantity, denoted by $x$, and its second attribute, quality, denoted by $s$. The second commodity is a numeraire good identified by its amount $y$, while this being its only attribute. The equilibrium price of the first commodity is $p$, whereas that of the numeraire is kept as unity. Below is the description of the consumer's problem under the framework (1) previously specified.

$$
\begin{aligned}
& \max _{x, s, y} U(x, s, y) \\
& \text { s.t. } B=\left(\begin{array}{ll}
x & y
\end{array}\right)\left(\begin{array}{l}
p \\
1
\end{array}\right), \quad\left(\begin{array}{lll}
x & s & y
\end{array}\right)=\left(\begin{array}{ll}
x & y
\end{array}\right)\left(\begin{array}{lll}
1 & a & 0 \\
0 & 0 & 1
\end{array}\right) .
\end{aligned}
$$

In the same manner, the firm's action (3) is described below:

$$
\max _{x, s, y}\left(\begin{array}{ll}
x & y
\end{array}\right)\left(\begin{array}{l}
p \\
1
\end{array}\right) \quad \text { s.t. } \quad F(x, s, y)=0, \quad s=G(x, y) .
$$


The first-order conditions for (6) and (7) together will be reduced:

$$
-\lambda \mu\left(\begin{array}{lll}
F_{x} & F_{s} & F_{y}
\end{array}\right)\left(\begin{array}{cc}
1 & 0 \\
G_{x} & G_{y} \\
0 & 1
\end{array}\right)=\left(\begin{array}{lll}
U_{x} & U_{s} & U_{y}
\end{array}\right)\left(\begin{array}{ll}
1 & 0 \\
a & 0 \\
0 & 1
\end{array}\right) .
$$

The non-spanning property of $\mathbf{G}_{\mathbf{x}}^{\prime}$ and $\mathbf{A}^{\prime}$ is obvious, while $0=G_{y}$ and $a=G_{x}$ are justified since $s=a x=G(x, y)$. Hence, we hereby rewrite the given structure (8) accordingly in a different fashion:

$$
\frac{U_{y}}{F_{y}}=\frac{U_{x}+a U_{s}}{F_{x}+a F_{s}}=-\lambda \mu \equiv \theta .
$$

The first-order conditions for (6) and (7) can alternatively be described in the following manner:

$$
\frac{U_{x}+a U_{s}}{U_{y}}=\frac{F_{x}+a F_{s}}{F_{y}}=p .
$$

These terms are however insufficient with respect to the first-order conditions for economic efficiency. Non-spanning is indeed the case when the economy fails to coordinate multiple items with a single price.

\section{Quality Adjustment Process}

Based on the above argument, we introduce a behavioral model of the producer who jointly produces quality. First, consider the gap between the WTP (willingness-topay), $\phi$, and the marginal cost, $\omega$, which is essentially zero at an equilibrium.

$$
\frac{U_{x}+a U_{s}}{U_{y}} \equiv \phi, \quad \frac{F_{x}+a F_{s}}{F_{y}} \equiv \omega .
$$

\section{Assumption (Base model)}

1. There is a producer called speculator in the member of the industry who challenges the commodity market by altering the quality of the commodity being produced.

2. The speculator will challenge the commodity market, not only with the quality, but also with the price that equals the marginal cost of the quality altered commodity. ${ }^{6}$

3. The consumers observe the quality and price of the speculator's commodity and decide if it is advantageous for them, and whether to alter their consumption by purchasing the quality altered commodity.

\footnotetext{
${ }^{6}$ The speculator is not a monopolist with the market power to dictate the price over the marginal cost; it is rather a marginal producer under competition as regards Assumption 5.
} 
Fig. 1 Marginal price of quality and the willingness to pay

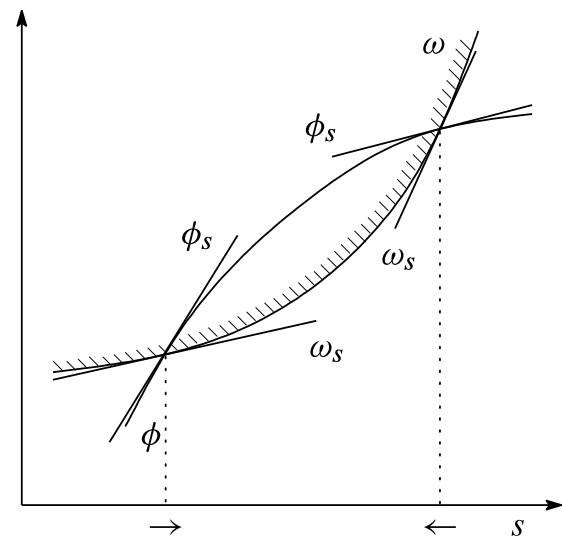

4. The consumers are variety neutral, that is, their assessment is only of the speculator's commodity and unaffected by other commodities.

5. If the new quality altered commodity is accepted, all the other firms will follow the speculator, and the market will again have homogeneous quality and price. If not, quality and price will remain unchanged.

Under the above assumptions, it is possible to infer the marginal price-quality assessments between supply and demand by observing the consequences of the consumer's accept or refuse decision without knowing the actual curvatures of $\phi$ and $\omega$, which cannot be observed.

As regards the market of commodity, the WTP, $\phi$, and the WTA (willingness-toaccept), which according to Assumption 2, equals the marginal cost, $\omega$, must always meet at an equilibrium, for any given quality. In addition, the commodity's marginal WTP with respect to quality, $\phi_{s}$, and the commodity's marginal WTA with respect to quality, $\omega_{s}$, should also meet at an equilibrium, according to the above mentioned assumptions. That is, if the speculator's challenge, $\Delta s$, conduces the commodity's WTP to exceed WTA, then the challenge will be approved; if not, the challenge will be disapproved. Illustrated in Fig. 1 is the typical situation, and according to which we may conjecture the following equivalences:

$$
\left\{\begin{array}{l}
\Delta s>0 \text { is accepted } \quad \Leftrightarrow \quad \phi_{s}-\omega_{s}>0, \\
\Delta s<0 \text { is accepted } \Leftrightarrow \phi_{s}-\omega_{s}<0 .
\end{array}\right.
$$

With respect to (12), quality adjustment can be expressed as the following differential equation, with $k>0$ being a velocity coefficient small enough to prevent overshooting:

$$
\phi_{s}-\omega_{s}=k \frac{\mathrm{d} s}{\mathrm{~d} t}=k \dot{s}
$$

At the stationary state $\dot{s}=0$, we have what follows with respect to (11):

$$
\frac{U_{x s}+a U_{s s}-p U_{y s}}{U_{y}}-\frac{F_{x s}+a F_{s s}-p F_{y s}}{F_{y}}=0 .
$$


As shown in the Appendix, from the above condition (13) and (9) taken into account, we arrive at the following first-order conditions for economic efficiency, which is essentially identical to (5):

$$
\frac{U_{y}}{F_{y}}=\frac{U_{s}}{F_{s}}=\frac{U_{x}}{F_{x}}=\theta .
$$

\section{Variety Averseness}

In this section, we consider the case in which the variety of commodity with respect to its quality matters to the consumer. In other words, we relax Assumption 4 of the basic model, and assume that consumers will be affected by not only the speculator's commodity, but also the remaining commodities. We denote the quality of the speculator's commodity by $\sigma$ and the consumer's WTP for the corresponding commodity by $\varphi$. The following quality adjustment process for the speculator is considered:

$$
\varphi_{\sigma}-\omega_{\sigma}=k \dot{\sigma}
$$

In this regard, we take $s=\sigma$ on the supply side for granted, with a speculator being one of many producing firms with identical technology. We therefore stay with $\omega$ to denote the marginal cost of producing commodities, and consequently perceive that $\omega_{\sigma}=\omega_{s}$; however, the willingness to pay $\phi$ is not same.

Since we assume that the speculator's commodity is differentiable (by its quality) from others, a consumer should have a varying willingness to pay upon the differentiated commodities. In that respect, the quality of the varied commodities, from the perspective of a variety conscious consumer, will be affected by the quality of the speculator's commodity as well as those of the other producers and the quantity of the varied commodities. What follows is how the consumer evaluates in effect the quality of the speculator's commodity: ${ }^{7}$

$$
s=\sigma+\eta V(\sigma, \xi, \bar{s}, \bar{x}) .
$$

The variety assessment function $V$ measures the variety of the commodities in terms of the entries, namely, the speculator's commodity $\sigma$, the quantity share of the speculator $\xi$, the quality of the remaining commodity $\bar{s}$, and the quantity share of the remaining commodity $\bar{x}$. Notice that $x=\xi+\bar{x}$ by definition. The consistence preference is accounted for by $\eta$ where a negative value designates variety averseness. The consumer's evaluation of the speculator's commodity therefore accounts for the quality of the corresponding commodity as well as the variety effect, as shown above.

In the following sections, we investigate how the consistency preference considered in (16) could affect the quality adjustment process specified in (15). In doing so,

\footnotetext{
${ }^{7}$ In this regard, we are implicitly assuming that a consumer evaluates quality differentiated commodities within the mixture of the commodities. For example, a firm's evaluation of an English peaking worker, with respect to the firm's productivity, will likely depend on the remaining workers' speaking language. Similar features can also be found in the bulky transactions of intermediate commodities.
} 
we assume for simplicity's sake that $U(x, s, y)=U(x, s)+y$ so that $\phi$ in (11) will be reduced simply to

$$
U_{x}+a U_{s}=\phi
$$

\subsection{Smooth Variety Assessment Function}

We first study the case in which the variety assessment function $V$ takes the form of variance i.e.,

$$
V(\sigma, \xi, \bar{s}, \bar{x})=\left(\sigma-\frac{\sigma \xi+\bar{s} \bar{x}}{\xi+\bar{x}}\right)^{2} \frac{\xi}{\xi+\bar{x}}+\left(\bar{s}-\frac{\sigma \xi+\bar{s} \bar{x}}{\xi+\bar{x}}\right)^{2} \frac{\bar{x}}{\xi+\bar{x}} .
$$

This is a smooth (quadratic) function, that is, the function is differentiable with respect to all variables including $\sigma$.

Let us calculate some of the derivatives beforehand:

$$
V_{\xi}=\frac{(\sigma-\bar{s})^{2}(\bar{x}-\xi) \bar{x}}{(\bar{x}+\xi)^{3}}, \quad V_{\sigma}=\frac{2(\sigma-\bar{s}) \xi \bar{x}}{(\bar{x}+\xi)^{2}} .
$$

We therefore have the following property for this smooth variety function at the homogeneous state, where $\sigma=\bar{s}$ :

$$
V(\bar{s}, \xi, \bar{s}, \bar{x})=V_{\xi}(\bar{s}, \xi, \bar{s}, \bar{x})=V_{\sigma}(\bar{s}, \xi, \bar{s}, \bar{x})=0 .
$$

Now investigate the demand schedule for the speculator's commodity $\varphi$ along with the simplification on the utility function made earlier in (17), such that $\varphi=U_{\xi}+a U_{\sigma}$ by definition. In the light of (16), what follows is evident:

$$
\varphi=U_{\xi}+a U_{\sigma}=U_{x}+\eta V_{\xi} U_{s}+\left(1+\eta V_{\sigma}\right) a U_{s} .
$$

According to (18), a smooth function such as (19) reduces to the following in the neighborhood of the homogeneous state:

$$
\varphi=U_{x}+a U_{s}=\phi
$$

Moreover, we may further differentiate to obtain the following:

$$
\varphi_{\sigma}=\left(U_{x s}+a U_{s s}\right)\left(1+\eta V_{\sigma}\right)=\phi_{s}\left(1+\eta V_{\sigma}\right)=\phi_{s} .
$$

Note that the last identity owes to (19). As shown below, we note that the dynamic characteristics of the quality adjustment process (15) in the neighborhood of the homogeneous state is essentially identical to the variety neutral case (12), which has been previously shown to satisfactorily meet first-order conditions for economic efficiency.

$$
k \dot{\sigma}=\varphi_{\sigma}-\omega_{\sigma}=\phi_{s}-\omega_{s} .
$$

In other words, adjustment process based on trial and error with variety averse utility and a smooth assessment of variety can be considered as approximately equivalent to the variety neutral case (Fig. 2). 
Fig. 2 Willingness to pay with smooth variety averseness

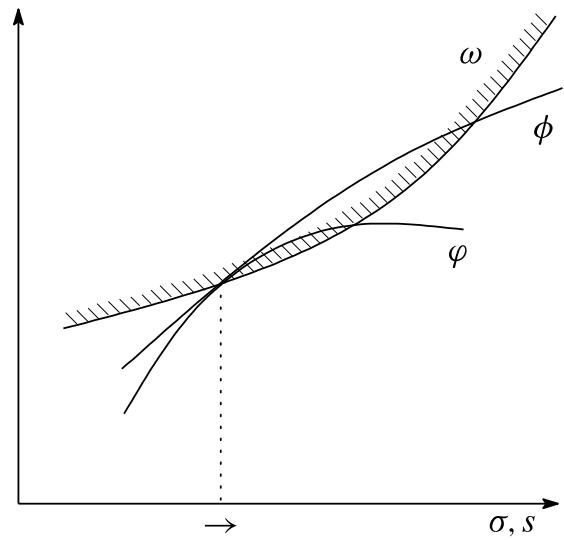

\subsection{Nonsmooth Variety Assessment Function}

Now, we suppose the variety assessment function takes the form of a standard deviation i.e.,

$$
V(\sigma, \xi, \bar{s}, \bar{x})=\sqrt{\left(\sigma-\frac{\sigma \xi+\bar{s} \bar{x}}{\xi+\bar{x}}\right)^{2} \frac{\xi}{\xi+\bar{x}}+\left(\bar{s}-\frac{\sigma \xi+\bar{s} \bar{x}}{\xi+\bar{x}}\right)^{2} \frac{\bar{x}}{\xi+\bar{x}}} .
$$

In this case, the derivatives are also calculated,

$$
V_{\xi}=\frac{(\bar{x}-\xi)(\sigma-\bar{s})}{2 \xi(\bar{x}+\xi)^{2}} \sqrt{\xi \bar{x}}, \quad V_{\sigma}=\frac{\sqrt{\xi \bar{x}}}{\bar{x}+\xi},
$$

while the property in a homogeneous state proves to be slightly different:

$$
V(\bar{s}, \xi, \bar{s}, \bar{x})=V_{\xi}(\bar{s}, \xi, \bar{s}, \bar{x})=0, \quad V_{\sigma}(\bar{s}, \xi, \bar{s}, \bar{x}) \neq 0 .
$$

Upon close examination of (20), we see that

$$
\lim _{\sigma \rightarrow \bar{s} \mp 0} V_{\sigma}=\mp \frac{\sqrt{\xi \bar{x}}}{\bar{x}+\xi} \neq 0, \quad V_{\sigma \sigma}=0 .
$$

Hence, in the neighborhood of the homogeneous state, we have

$$
\varphi_{\sigma}=U_{x s}\left(1+\eta V_{\sigma}\right)+a U_{s s}\left(1+\eta V_{\sigma}\right)^{2} \neq \phi_{s}
$$

according to (21) and (22).

With respect to (23) concerns about the adjustment process might be raised. Note that $\varphi_{\sigma}$ can be larger than $\phi_{s}$ when $\sigma$ approaches $\bar{s}$ from below (left) according to (22), because $\eta$ is negative for a variety averse consumer. At the same time, $\varphi_{\sigma}$ becomes smaller and even negative when $\sigma$ approaches from above (right) for the very same reason. In such cases, the adjustment process will stop at a homogeneous and possibly inefficient state, despite the speculator's continuous exploration of different 
Fig. 3 Willingness to pay with non-smooth variety averseness

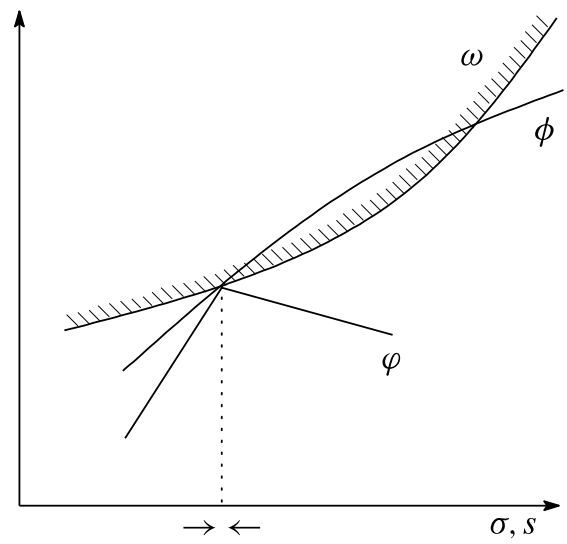

qualities. Such a situation is described below and also illustrated in Fig. 3. The case is further demonstrated using a simple general equilibrium model of recycling in the next section.

$$
\left.\begin{array}{l}
\phi_{s}-\omega_{s}<\varphi_{\sigma}-\omega_{\sigma}=k \dot{\sigma}>0(\sigma<\bar{s}) \\
\phi_{s}-\omega_{s}>\varphi_{\sigma}-\omega_{\sigma}=k \dot{\sigma}<0(\sigma>\bar{s})
\end{array}\right\} \Rightarrow k \dot{\sigma}=0(\sigma=\bar{s}) .
$$

\subsection{Unidentifiable Case}

Thus far, we have assumed that the speculator's commodity is at least differentiable from others. We now relax this assumption. Since we assume the speculator's commodity is unidentifiable to the consumer, the consumer's assessment of quality will not be of the producer's particular product, but rather of the blended bulk of all the products. For the sake of simplicity, we assume that the assessment of quality is represented by the quantity-weighted quality and the magnitude of variety is ignored:

$$
s=\frac{\sigma \xi+\bar{s} \bar{x}}{\xi+\bar{x}}=\frac{1}{n} \sigma+\left(1-\frac{1}{n}\right) \bar{x} .
$$

Notice that $1 / n$ designates the fraction of the speculator's market share.

The willingness to pay for the undifferentiable products (in this case, we write it as $\phi$ because the commodities are homogenized) is as defined in (17) because the quality assessment is $s$. But when the construction of $s$ is what is as described in (24), the differential upon $\phi$ will be affected:

$$
\phi_{\sigma}=\frac{1}{n}\left(U_{x s}+a U_{s S}\right)=\frac{1}{n} \phi_{s} .
$$

We illustrate the possible status in Fig. 4. As the share of the speculator becomes insignificant, that is, as $1 / n$ gets smaller, $\phi_{\sigma}$ gets smaller if not negative. In this regard, the speculator may alter the quality of the product $\sigma$ in the opposite direction, and the quality $s$ will eventually settle at a state that is inefficient, such that 
Fig. 4 Non-identifiable case

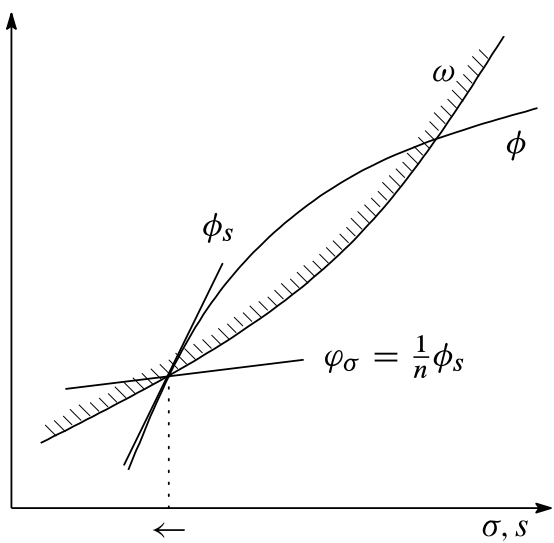

Fig. 5 Recycling economy

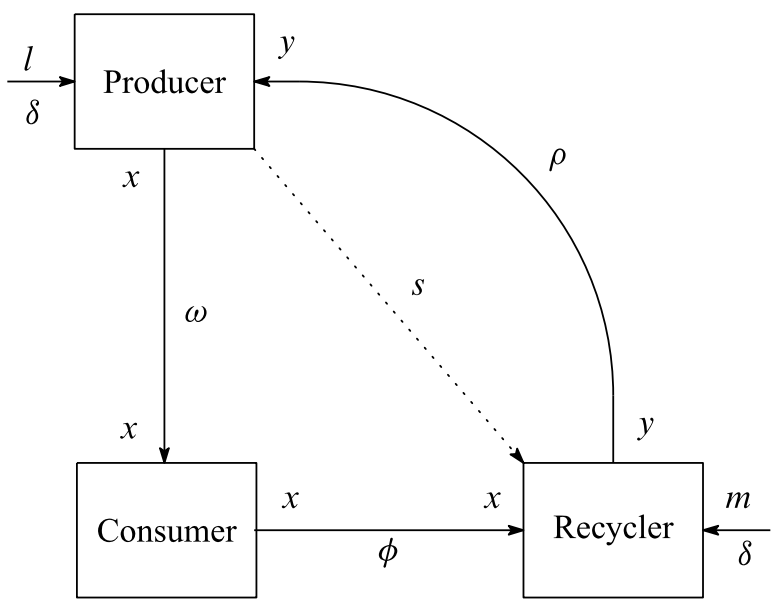

$\frac{1}{n} \phi_{s}=\omega_{\sigma}$. Finally, we note that the variety averse issues discussed in the earlier sections can also arise in the unidentifiable case, although we do not further delve into this matter.

\section{Example: Recycling Economy}

\subsection{Brief Description of the Model}

The core elements of the model are adopted from Nishimura (2008). All notations on variables are therefore subject to the original paper. There are three agents in the recycling market: the producer, consumer, and recycler (Fig. 5). The producer jointly produces the final commodity of amount $x$, and its quality (recyclability) whose quantified amount is $s$, by using inputs, the secondary material of amount $y$, and the labor of amount $l$. For simulation purposes, we study the following production functions:

$$
x=y^{\alpha} l^{1-\alpha}, \quad s=y^{-\alpha} l^{\alpha} .
$$


The recycler produces the secondary material of amount $y$ by using inputs, namely the waste that comes from the consumer, whose amount coincides with that of the primary production $x$, and the labor amount $m$. We use the following example as the recycler's production function:

$$
y=x^{\beta} m^{1-\beta} s^{\epsilon} .
$$

The market is evaluated by the consumer's utility, whose utility is simply ruled by the consumer's quantity of consumption of the commodity, which is measured by $x$. The model is closed by giving the labor resource constraint that amounts to $Q$, such that $l+m \leq Q$.

Given the market prices, we describe the behavior of each agent. The market price for the commodity, the secondary material, and the labor be denoted $\omega, \rho$, and $\delta$, respectively. The recycler's willingness to pay is denoted by $\phi$. No price will be levied on the recyclability due to the underlying non-spanning assumption. The producer's problem is therefore to solve

$$
\max _{x, y, l} \omega x-\rho y-\delta l \quad \text { s.t. }
$$

for which we have the following first-order condition:

$$
\omega=\rho s^{1-\alpha}+\delta s .
$$

In the same vein, the recycler's problem is to solve

$$
\max _{x, y, m} \rho y-\phi x-\delta m \quad \text { s.t. }
$$

and the first-order condition follows:

$$
\phi=s^{\epsilon / \beta} \beta(1-\beta)^{(1-\beta) / \beta} \delta^{(\beta-1) / \beta} \rho^{1 / \beta} .
$$

Due to the nature of the consumer's utility, $\omega=\phi$ must hold at the equilibrium for any given $s$. In this case, we may solve for $\rho$ with respect to (28) and (29). The solution can be written $\rho(s)$ to indicate that $\rho$ is subject to $s$. Now the ceteris paribus price of the commodity with respect to its recyclability $s$, with other things (including $\rho$ ) being equal, becomes

$$
\omega(s ; \bar{s})=\rho(\bar{s}) s^{1-\alpha}+\delta s
$$

where $\bar{s}$ is the underlying recyclability employed by the remaining producers. In a similar manner, the ceteris paribus willingness to pay of the recycler to pay for the wastes with respect to the recyclability $s$ of the corresponding commodity, with other things being equal, follows below:

$$
\phi(s ; \bar{s})=s^{\epsilon / \beta} \beta(1-\beta)^{(1-\beta) / \beta} \delta^{(\beta-1) / \beta}(\rho(\bar{s}))^{1 / \beta} .
$$

The equilibrium net price of homogeneous commodity with respect to its recyclability is denoted by $\pi(s)$, while by definition, we note that

$$
\pi(s)=\omega(s ; s)-\phi(s ; s) .
$$


Table 1 Parameters in simulation cases

\begin{tabular}{|c|c|c|c|c|c|c|c|c|c|}
\hline & $\alpha$ & $\beta$ & $\epsilon$ & $\delta$ & $Q$ & $s^{*}$ & $\eta$ & $v$ & $n$ \\
\hline Case 1 & 0.5 & 0.2 & 0.3 & 5 & 20 & 1.2 & 0 & - & - \\
\hline Case 2 & & & & & & & -10 & 1 & 100 \\
\hline Case 3 & & & & & & & -0.2 & 0.5 & 100 \\
\hline
\end{tabular}

\subsection{Speculator and Variety Averseness}

Now introduce the speculator designated in Sect. 3. The speculator's recyclability is denoted by $\sigma$. As the speculator is one of many with identical production technology, the commodity price of the speculator will be those specified in (30), except the recyclability is of the speculator:

$$
\omega(\sigma ; \bar{s})=\rho(\bar{s}) \sigma^{1-\alpha}+\delta \sigma
$$

On the other hand, the recycler's willingness to pay for the speculator's identifiable commodity/waste will depend on the recycler's assessment of its recyclability, that is,

$$
\varphi(\sigma ; \bar{s})=(s(\sigma, \bar{s}))^{\epsilon / \beta} \beta(1-\beta)^{(1-\beta) / \beta} \delta^{(\beta-1) / \beta}(\rho(\bar{s}))^{1 / \beta} .
$$

The recycler's assessment of recyclability $s(\sigma, \bar{s})$ depends on the variety aversion $\eta$ and the measure of variability:

$$
s(\sigma, \bar{s})=\sigma+\eta\left(\left(\sigma-\frac{\sigma \xi+\bar{s} \bar{x}}{\xi+\bar{x}}\right)^{2} \frac{\xi}{\xi+\bar{x}}+\left(\bar{s}-\frac{\sigma \xi+\bar{s} \bar{x}}{\xi+\bar{x}}\right)^{2} \frac{\bar{x}}{\xi+\bar{x}}\right)^{v}
$$

where $v$ characterizes the form of variety assessment function such as variance $(v=1)$ and standard deviation $(v=0.5)$. Notice that $\xi$ and $\bar{x}$ are the quantitative share of the speculator and the rest of the producers, respectively. The quality (recyclability) adjustment process in this case can be described as the net price-minimizing behavior of the speculator. The process described below will find at least a local minimum.

$$
-\left(\omega_{\sigma}-\varphi_{\sigma}\right)=k \dot{\sigma}, \quad k>0 .
$$

Note that this process is essentially identical to the previous function (15).

We examine the corresponding dynamic process using pro forma numbers shown in Table 1. Figures 6, 7, 8, illustrate the mutatis mutandis (as opposite to ceteris paribus) net price $\pi(s)$ for the indicated interval of $s$. The $s$ that minimizes $\pi(s)$ is denoted $s^{*}$ and the value is shown in the table. In all simulation cases, the underlying recyclability is the same, that is, $\bar{s}=1.1$. Case 1 represents the variety neutral case, in which the recycler's assessment of speculator's commodity/waste is unaffected by those of the remaining producers such that $s(\sigma ; \bar{s})=\sigma$. We can conclude from Fig. 6 that in the neighborhood of $\bar{s}$ the descent direction of the ceteris paribus net price $\omega-\varphi$ is oriented toward the desired $s^{*}$. 


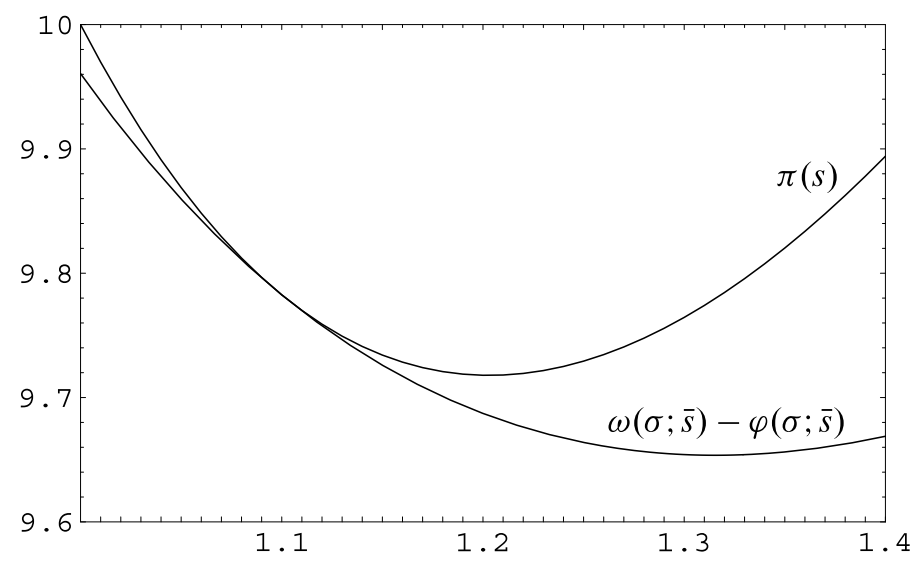

Fig. 6 Speculator's ceteris paribus net price at $\bar{s}=1.1$, in case 1

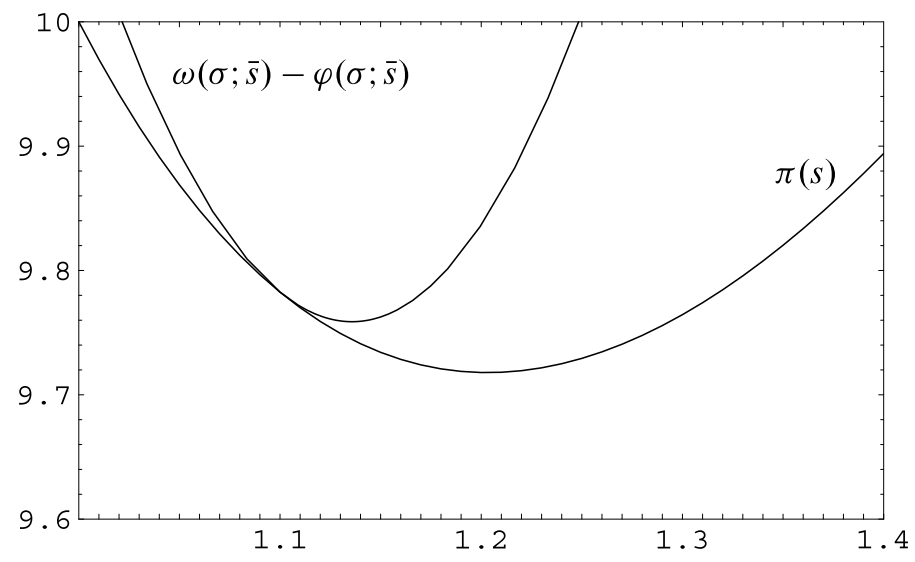

Fig. 7 Speculator's ceteris paribus net price at $\bar{s}=1.1$, in case 2

Figure 7 illustrates case 2 when the recycler is variety averse $(\eta<0)$, while the recycler's variety assessment is with a smooth function $(v=1)$. As illustrated, the descent direction of the ceteris paribus net price is the same as that of the mutatis mutandis net price $\pi$ which is destined to $s^{*}$, in the neighborhood of $\bar{s}$. It can also be noted that this is true for any $\eta<0$, provided the variety assessment function is smooth $(v=1)$. The non-smooth case, $v=0.5$, is illustrated in Fig. 8. The descent direction of the ceteris paribus net price in this case is oriented not toward $s^{*}$ but toward $\bar{s}$, indicating that the recyclability adjusting process will fail to achieve the socially preferred state. 


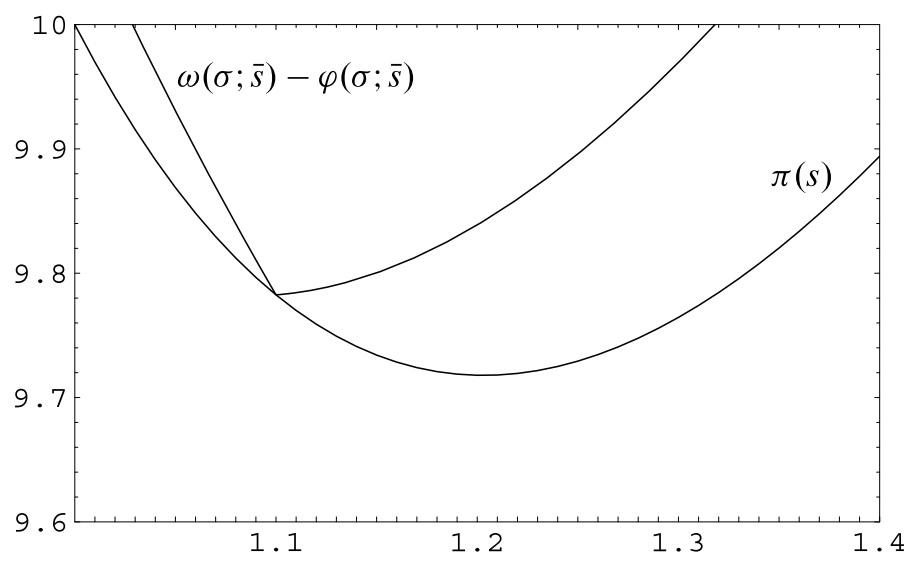

Fig. 8 Speculator's ceteris paribus net price at $\bar{s}=1.1$, in case 3

\section{Concluding Remarks}

The Japanese saying "the pile that sticks out gets hammered down," essentially means that a person who stands out too much is subject to reprisal (or assimilation). In our model, we found that a producer seeking the opportunity to ameliorate its product can face reprisal due to the non-smooth variety aversion of the consumer. At the same time, we also found that this is not the case if the variety assessment is with a smooth function and the speculator's strategy will lead to a state of efficiency.

The quality adjustment of producers based on trial and error discussed in this paper can be relevant when the economy lacks a sufficient number of commodity markets for all the attributes (and therefore quality) to be coordinated indirectly. Such a nonspanning structure can be typical in the markets of intermediate commodities with many inseparable attributes. Contrasted with final products purchased by end consumers with different tastes and a preference for variety, the market for intermediate commodities tends to contract as inputs of different quality are often perfect substitutes in production.

Producers, perhaps through research and development, change the quality of their product in pursuit of prosperity on the basis of trial and error. A consumer's implicit valuation of the attributes are learned by the speculator and, eventually, by all providers through the consistent quality-price movement of the commodity. The implicit value of attributes, although not quite as efficient as the Lindahl price, is the necessary information for achieving economic efficiency. A quality adjusting producer and a variety averse consumer are perhaps the ones that are hammering out the technological structures of the economy.

\section{Competing Interests}

The author declares that he has no competing interests. 
Acknowledgements The author would like to thank the anonymous reviewers for their valuable comments and suggestions to improve the quality of the paper.

\section{Appendix}

By re-arranging (13), we have

$$
\frac{U_{x s}+a U_{s s}-p U_{y s}}{F_{x s}+a F_{s s}-p F_{y s}}=\frac{U_{y}}{F_{y}}=\theta
$$

where the last identity holds for the sake of (9). Hence, should there be some $\delta \neq 0$ such that

$$
U_{x s}+a U_{s s}-p U_{y s}+\delta U_{y}=F_{x s}+a F_{s s}-p F_{y s}+\delta F_{y}=0
$$

so that we may apply l'Hôspital's rule with respect to $p$ in the following formula to obtain the first identity below. The remaining identities are due to (31).

$$
\frac{U_{x s}+a U_{s s}-p U_{y s}+\delta U_{y}}{F_{x s}+a F_{s s}-p F_{y s}+\delta F_{y}}=\frac{U_{y s}}{F_{y s}}=\frac{U_{y}}{F_{y}}=\theta .
$$

Meanwhile, we may find $\varepsilon \neq 0$ such that

$$
U(x, y, s)-\varepsilon=\theta(F(x, y, s)-\varepsilon),
$$

so that for some $\gamma \neq 0$, we have

$$
U(x, y, s)-\varepsilon+\gamma U_{y}=F(x, y, s)-\varepsilon+\gamma F_{y}=0 .
$$

This enables l'Hôspital's rule again, but this time with respect to $s$, thus obtaining

$$
\frac{U(x, y, s)-\varepsilon+\gamma U_{y}}{F(x, y, s)-\varepsilon+\gamma F_{y}}=\frac{U_{s}+\gamma U_{y s}}{F_{s}+\gamma F_{y s}}=\frac{U_{s}}{F_{s}}=\theta
$$

where the last identity holds because of (32). Finally, (34), (13), and (9) gives the wanted result (14).

\section{References}

Adner R, Levinthal D (2001) Demand heterogeneity and technology evolution: implications for product and process innovation. Manag Sci 47(5):611-628. doi:10.1287/mnsc.47.5.611.10482

Batten D, Boschetti F (2008) Visions of evolution: self-organization proposes what natural selection disposes. Biol Theory 3:17-29. doi:10.1162/biot.2008.3.1.17

Brun A, Pero M (2012) Measuring variety reduction along the supply chain: the variety gap model. Int J Prod Econ 139(2):510-524. doi:10.1016/j.ijpe.2012.05.018

Callander S (2011) Searching and learning by trial and error. Am Econ Rev 101(6):2277-2308. doi:10.1257/aer.101.6.2277

Dreze JH, Hagen KP (1978) Choice of product quality: equilibrium and efficiency. Econometrica 46(3):493-513 
Economides N (1984) The principle of minimum differentiation revisited. Eur Econ Rev 24(3):345-368

Hosoda EB (2012) Recycling of waste and downgrading of secondary resources in a classical type of production model. J Econ Struct 1:7. doi:10.1186/2193-2409-1-7

Hotelling H (1929) Stability in competition. Econ J 39(153):41-57

Jwa SH (1985) Perfect competition and quality variation. Korean Econ Rev 1(1):15-37

Kaiser K (2011) Variety aversion and information overload: an experimental approach. Working paper 2011-01, Banco de Mexico

Lancaster KJ (1966) A new approach to consumer theory. J Polit Econ 74:132-157

Leland HE (1977) Quality choice and competition. Am Econ Rev 67(2):127-137

Levav J, Heitmann M, Herrmann A, Iyengar SS (2010) Order in product customization decisions: evidence from field experiments. J Polit Econ 118(2):274-299. doi:10.1086/652463

McFadden D (1986) The choice theory approach to market research. Mark Sci 5(4):275-297. doi: $10.1287 / \mathrm{mksc} .5 .4 .275$

Nishimura K (2008) The role and internalization of homogeneous and non-homogeneous design effects in recycling systems. Metroeconomica 59(2):110-130. doi:10.1111/j.1467-999X.2008.00295.X

Rosen S (1974) Hedonic prices and implicit markets: product differentiation in pure competition. J Polit Econ 82(1):34-55

Sato K (2012) Nonmyopia and incentives in the piecewise linearized MDP procedures with variable stepsizes. J Econ Struct 1:5. doi:10.1186/2193-2409-1-5

Shaked A, Sutton J (1987) Product differentiation and industrial structure. J Ind Econ 36(2):131-146 